\title{
Further Investigation of Massive Landau-Gauge Propagators in the Infrared Limit
}

\author{
Attilio Cucchieri and Tereza Mendes* \\ IFSC, University of São Paulo, C.P. 369, CEP 13560-970, São Carlos SP, Brazil. \\ E-mail: attiliodifsc.usp.br, mendes@ifsc.usp.br
}

\begin{abstract}
We investigate how the infrared behavior of electric and magnetic gluon propagators in Landau gauge is affected by temperature. More precisely, we perform large-lattice simulations in pure $\mathrm{SU}(2)$ gauge theory around the transition temperature $T_{c}$ and study the longitudinal (electric) and transverse (magnetic) gluon propagators in momentum space, proposing the calculation of screening masses through an Ansatz from the zero-temperature case. Going from zero to nonzero temperature, we see that the longitudinal gluon propagator $D_{L}(p)$ is enhanced, with an apparent plateau value in the infrared, while the transverse propagator $D_{T}(p)$ gets progressively more infrared-suppressed, with a clear turnover in momentum at all nonzero temperatures considered. Our data allow us to associate what was previously seen as a peak in the infrared value of $D_{L}(p)$ at $T_{c}$ to severe finite-size effects along the temperature direction. In particular, a temporal lattice extent $N_{t} \geq 8$ seems to be needed to study the electric sector around the transition. Once these systematic errors are eliminated, the infrared behavior of the longitudinal propagator appears to be rather independent of the temperature below the transition. Above $T_{c}$, the infrared value of $D_{L}(p)$ starts to decrease monotonically with the temperature.
\end{abstract}

The XXVIII International Symposium on Lattice Field Theory, Lattice2010

June 14-19, 2010

Villasimius, Italy

\footnotetext{
* Speaker.
} 


\section{Introduction}

At high temperatures, deconfinement is expected to be felt in the long-distance behavior of correlation functions - such as the (real-space) longitudinal gluon propagator - as an exponential falloff with the distance, defining a screening length and conversely a screening mass [1]. Although this predicted behavior has been established at high temperatures down to around twice the critical temperature $T_{c}$ [2], it is not clear how a screening mass would show up around $T_{c}$. At the same time, recent studies of zero-temperature Landau-gauge propagators on large lattices have shown a (dynamical) gluon mass at $T=0$ (see e.g. [3] for a discussion), in agreement with the so-called massive solution of Schwinger-Dyson equations [4]. One can try to use this knowledge to define temperature-dependent masses for the region around the critical temperature. In the following, we review briefly the lattice results for the zero-temperature case, discuss the expected behavior for nonzero temperature and show our preliminary results for the gluon propagator on large lattices for several values of the temperature, drawing our conclusions. A more detailed analysis and additional data will be presented elsewhere [5].

\section{Expected Behavior}

At zero temperature, Landau-gauge gluon and ghost propagators are expected to behave according to the so-called Gribov-Zwanziger scenario, which is based on restricting the gauge configurations to the region delimited by the first Gribov horizon, where the smallest nonzero eigenvalue of the Faddeev-Popov matrix goes to zero [6]. Essentially, the infinite-volume limit favors configurations on the first Gribov horizon and, as a result, the ghost propagator should become infrared-enhanced, inducing long-range effects in the theory. (In Coulomb gauge, the restriction to the first Gribov region causes the appearance of a confining color-Coulomb potential.) In this scenario, formulated for momentum-space propagators, the long-range features needed to explain the color-confinement mechanism are thus manifest in the ghost propagator, whereas the momentumspace gluon propagator $D(p)$ is suppressed in the infrared limit. Such a suppression is associated with violation of spectral positivity, which is commonly regarded as an indication of gluon confinement. In fact, $D(0)$ is originally expected to be zero, corresponding to maximal violation of spectral positivity. The parametrization of this behavior as a propagator with a pair of poles with conjugate complex masses was proposed by Gribov, in connection with his study of gauge copies.

Lattice studies have confirmed the suppression of the gluon propagator in the infrared limit and the enhancement of the ghost propagator at intermediate momenta. However, once the investigated lattice sizes were large enough, it became clear that the standard procedure for gauge fixing and simulations is not compatible with the original scenario in the deep infrared regime. Indeed, the gluon propagator attains a finite value as the momentum is taken to zero and the enhancement of the ghost propagator does not persist in this limit. We note the very large lattice sizes employed in order to observe such a behavior, $L \approx 20 \mathrm{fm}$ and larger. The status of these zero-temperature simulations has been recently reviewed in [7]. A good fit of the massive behavior for the gluon propagator is obtained from the Gribov-Stingl form, which generalizes the Gribov form described above (see e.g. [8]). In any case, violation of reflection positivity for the real-space gluon propagator is observed for all cases studied [9]. 
As the temperature $T$ is turned on, one expects to observe Debye screening of the color charge, signaled by screening masses/lengths that can in principle be obtained from the gluon propagator [10]. More specifically, chromoelectric (resp. chromomagnetic) screening will be related to the longitudinal (resp. transverse) gluon propagator computed at momenta with null temporal component, i.e. with $p_{0}=0$ (soft modes). At high temperatures, we expect the real-space longitudinal propagator to fall off exponentially at long distances, defining a (real) electric screening mass, which can be calculated perturbatively to leading order. Also, according to the $3 \mathrm{~d}$ adjoint-Higgs picture for dimensional reduction, we expect the transverse propagator to show a confining behavior at finite temperature, in association with a nontrivial magnetic mass (see e.g. [2]). The ghost propagator, on the other hand, should not depend on $T$. We note that these propagators are gauge-dependent quantities, and the (perturbative) prediction that the propagator poles might be gauge-independent must be checked, by considering different gauges.

The above expectations have been checked and confirmed for the gluon propagator at high $T$ for various gauges $[2,11]$. The behavior of Landau-gauge gluon and ghost propagators around the critical temperature $T_{c}$ has been investigated in [12]. The study showed a stronger infrared suppression for the transverse propagator than for the longitudinal one, confirming the dimensionalreduction picture also at smaller temperatures. [We note here that a very recent study [13] discusses whether this suppression is consistent with $D_{T}(0)=0$ and investigates Gribov-copy effects for the propagators.] It was also found that the ghost propagator is insensitive to the temperature, as predicted. For the longitudinal gluon propagator, a very interesting behavior was seen: the data approach a plateau (as a function of the momentum) in the infrared region and, as a function of temperature, this plateau shows a sharp peak around the critical temperature. The exact behavior around $T_{c}$ (e.g. whether the peak turns into a divergence at infinite volume) could not be determined, since relatively small lattices were used. All studies mentioned so far are for SU(2) gauge theory. The momentum-space expressions for the transverse and longitudinal gluon propagators $D_{T}(p)$ and $D_{L}(p)$ can be found e.g. in [12].

Recently, in [14], further simulations around $T_{c}$ confirmed the above results, and lattice data for the gluon propagator were used to construct an order parameter for the chiral/deconfinement transition. More precisely, the authors use a much finer resolution around $T_{c}$ and consider the $\mathrm{SU}(2)$ and $\mathrm{SU}(3)$ cases. A check of their calculation is done for the electric screening mass, taken as $D_{L}(0)^{-1 / 2}$ and extracted from the data, where only the $p=0$ raw data point is used. The considered lattice sizes are still moderate.

Of course, even if an exponential fit to the longitudinal gluon propagator works at high temperature, it is not obvious that this should hold at $T \gtrsim T_{c}$. One should therefore consider more general fits. At $T=0$, the momentum-space propagator is well fitted by a Gribov-Stingl form (see e.g. [8]), allowing for complex-conjugate poles

$$
D_{L, T}(p)=C \frac{1+d p^{2 \eta}}{\left(p^{2}+a\right)^{2}+b^{2}} .
$$

This expression corresponds to two poles, at masses $m^{2}=a \pm i b$, where $m=m_{R}+i m_{I}$. The mass $m$ thus depends only on $a, b$ and not on the normalization $C$. The parameter $\eta$ should be 1 if the fitting form also describes the large momenta region (from our infrared data we get $\eta \neq 1$ ). For consistency with the usual definition of electric screening mass, we expect to observe $m_{I} \rightarrow 0$ 
$(b \rightarrow 0)$ for the longitudinal gluon propagator at high temperature. Clearly, if the propagator has the above form, then the screening mass defined by $D_{L}(0)^{-1 / 2}=\sqrt{\left(a^{2}+b^{2}\right) / C}$ mixes the complex and imaginary masses $m_{R}$ and $m_{I}$ and depends on the (a priori arbitrary) normalization $C$.

\section{Results}

We have considered the pure SU(2) case, with a standard Wilson action. For our runs we employ a cold start, performing a projection on positive Polyakov loop configurations. Also, gauge fixing is done using stochastic overrelaxation and the gluon dressing functions are normalized to 1 at $2 \mathrm{GeV}$. We take $\beta$ values in the scaling region and lattice sizes ranging from $N_{s}=48$ to 192 and from $N_{t}=2$ to 16 lattice points, respectively along the spatial and along the temporal directions. The temperature is given by $T=1 / N_{t} a$.

All our data have been fitted to a Gribov-Stingl behavior, as described in the previous section (see Eq. 2.1). These fits are shown below in all plots, whereas a detailed discussion of the associated masses $m_{R}, m_{I}$ will be presented elsewhere [5]. We generally find good fits to the Gribov-Stingl form (including the full range of momenta), with nonzero real and imaginary parts of the pole masses in all cases. For the transverse propagator $D_{T}(p)$, the masses $m_{R}$ and $m_{I}$ are of comparable size. The same holds for $D_{L}(p)$, but in this case the relative size of the imaginary mass seems to decrease with increasing temperature. We also looked at the real-space propagators, finding clear violation of reflection positivity for the transverse propagator at all temperatures. For the longitudinal propagator, posivity violation is observed unequivocally only at zero temperature and for a few cases around the critical region, in association with the severe systematic errors discussed below.

Our runs were initially planned under the assumption that a temporal extent $N_{t}=4$ might be sufficient to observe the infrared behavior of the propagators. (Our goal was, then, to increase $N_{s}$ significantly, to check for finite-size effects.) For this value of $N_{t}$, the chosen $\beta$ values: 2.2872, $2.299,2.313,2.333,2.505796$ yield temperatures respectively of $0.968,1.0,1.04,1.1,1.936$ times the critical temperature $T_{c}$. As is clear from Fig. 1 below, the assumption that $N_{t}=4$ might be enough is not verified for the longitudinal propagator around the critical temperature, especially in the case of larger $N_{s}$.

Indeed, as $N_{s}$ is doubled from 48 to 96 and then to 192, we see that the infrared value of $D_{L}(p)$ changes drastically, resulting in a qualitatively different curve at $N_{s}=192$, apparently with a turnover in momentum. Also, in this case the real-space longitudinal propagator manifestly violates reflection positivity. We took this as an indication that our choice of $N_{t}=4$ was not valid and therefore considered larger values of $N_{t}$, obtaining a clearer picture of the critical behavior of $D_{L}(p)$. As seen in Fig. 1 (top), once we use large enough values of $N_{t}$, the curve stabilizes (within statistical errors) for four different combinations of parameters. In particular, the two curves at fixed physical volume (the yellow and the gray curves) agree very nicely. Note also that the (orange) curve corresponding to the smallest physical spatial size (i.e. $4 \mathrm{fm}$ ), may show mild finite-size effects. As seen in the bottom plot, the finite-physical-size effects are more pronounced for the transverse propagator, which does not seem to suffer from the same small- $N_{t}$ effects. In this case, we see clearly the strong infrared suppression of the propagator, with a turnover at around 400 $\mathrm{MeV}$. 

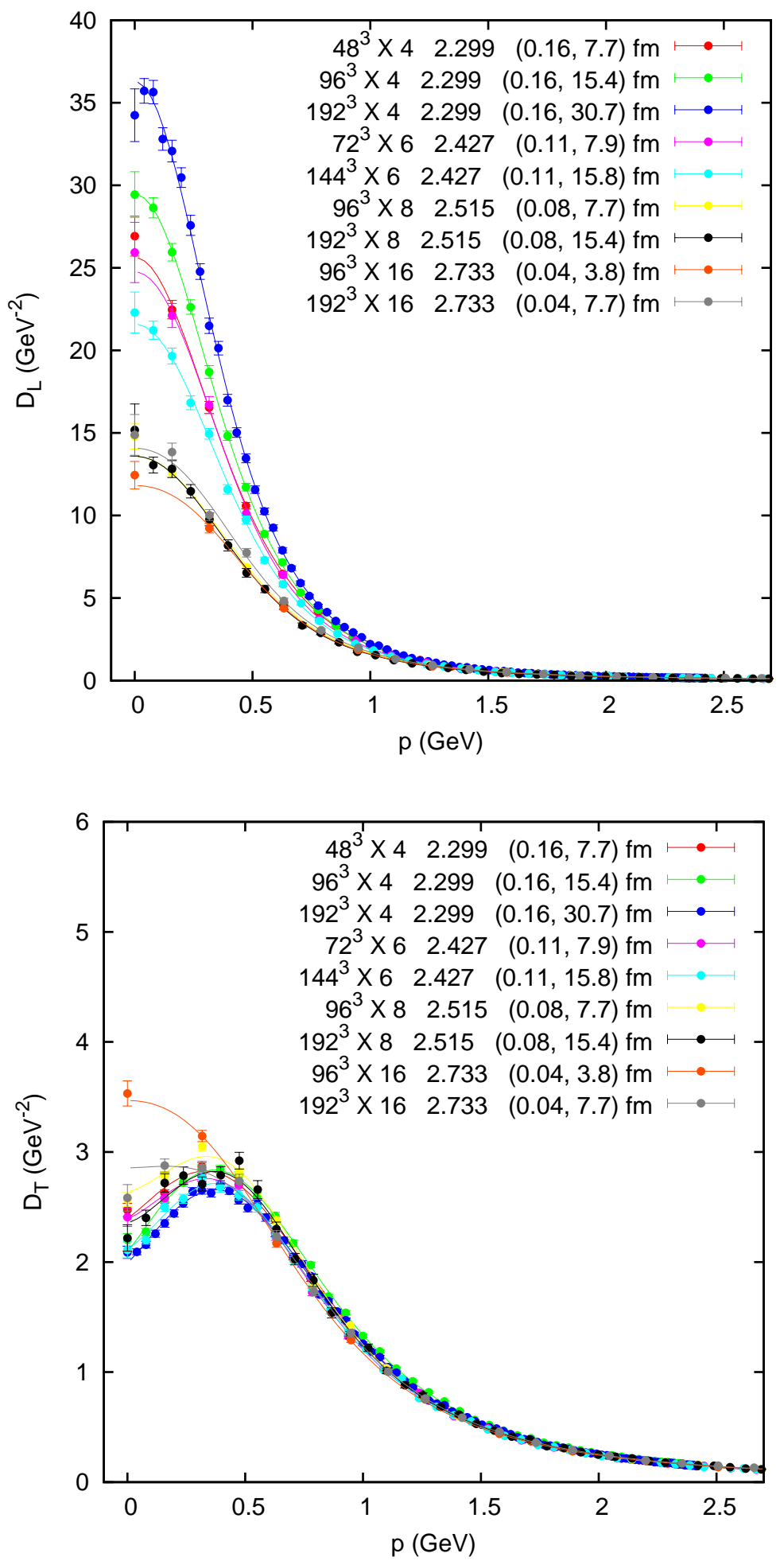

Figure 1: Longitudinal (top) and transverse (bottom) gluon propagator at $T_{c}$, for various lattice sizes and values of $\beta$. Values for $N_{s}^{3} \times N_{t}, \beta$, lattice spacing $a$ and spatial lattice size $L$ (both in $\mathrm{fm}$, in parentheses) are given in the plot labels. The resulting temperature is about $302 \mathrm{MeV}$. 

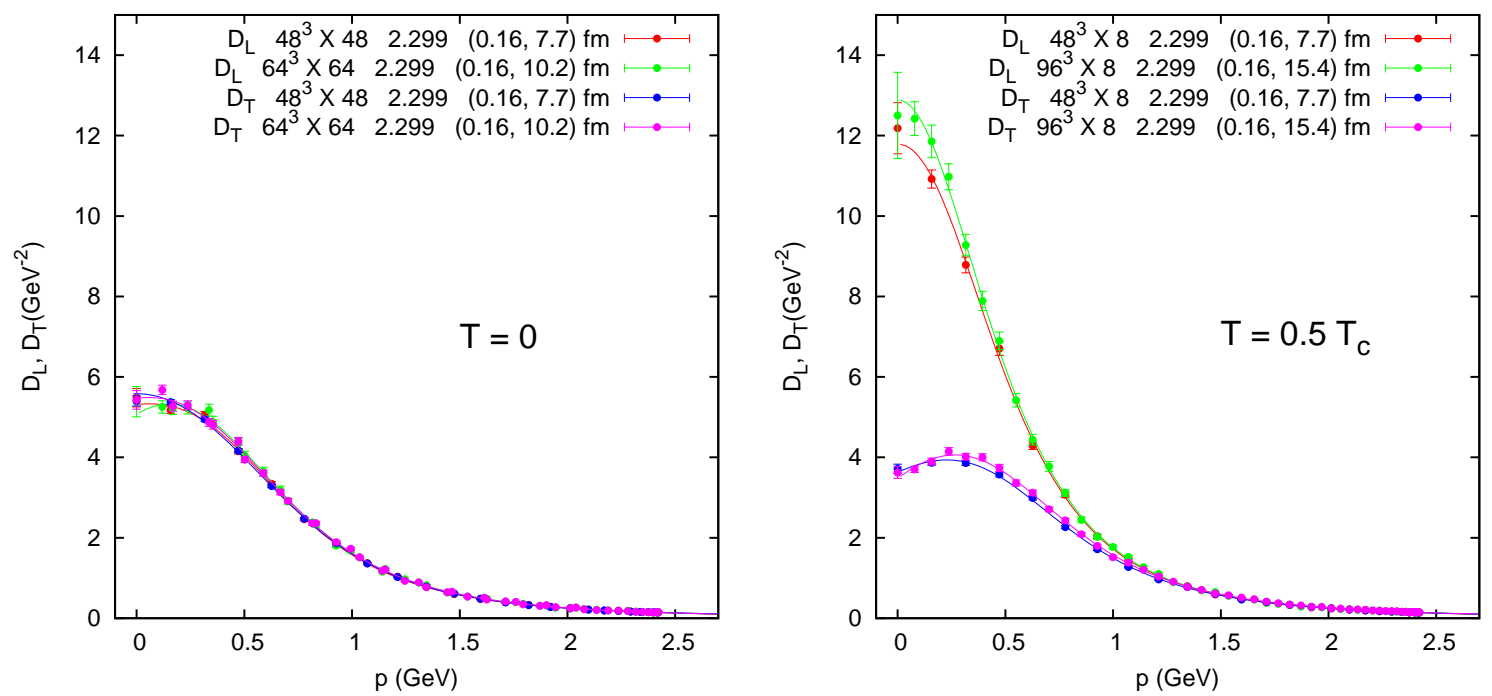

Figure 2: Longitudinal and transverse gluon propagators at $T=0$ (left) and $T=T_{c} / 2$ (right). Values for $N_{s}^{3} \times N_{t}, \beta$, lattice spacing $a$ and spatial lattice size $L$ (both in fm, in parentheses) are given in the plot labels.
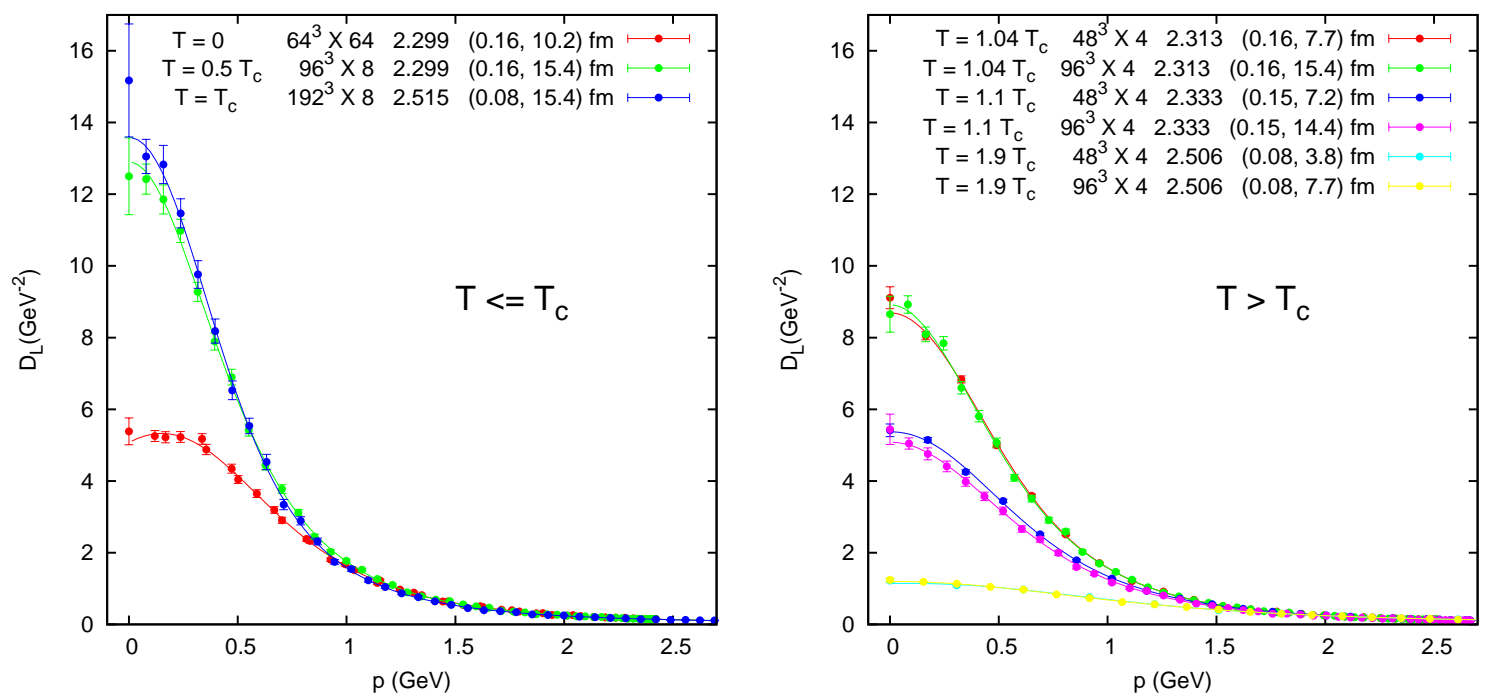

Figure 3: Longitudinal gluon propagator for $T \leq T_{c}$ (left) and $T>T_{c}$ (right). Values for $N_{s}^{3} \times N_{t}, \beta$, lattice spacing $a$ and spatial lattice size $L$ (both in fm, in parentheses) are given in the plot labels.

The above systematic errors were not observed for other simulated values of the temperature (except for the data at $0.968 T_{c}$, not shown here), including the temperature values just above $T_{c}$. We also did runs at $T=0$ and $T \approx T_{c} / 2$, for which we show combined $D_{L}(p)$ and $D_{T}(p)$ data in Fig. 2. We see a jump in $D_{L}(p)$ as temperature is switched on, while $D_{T}(p)$ decreases slightly, showing a clear turnover point at around $350 \mathrm{MeV}$. In Fig. 3 we collect data for $D_{L}(p)$ at several temperatures (for $T \leq T_{c}$, for clarity, we show only valid lattices with the largest physical size). Note that the curve remains unchanged (within errors) from $T_{c} / 2$ to $T_{c}$. Above $T_{c}$, there is a steady drop. For all values of $T, D_{L}(p)$ seems to reach a plateau at small $p$. 


\section{Conclusions}

The transverse gluon propagator $D_{T}(p)$ shows infrared suppression and a turnover in momentum (in agreement with the dimensional-reduction picture) at all nonzero temperatures considered. The longitudinal propagator $D_{L}(p)$, on the contrary, appears to reach a plateau at small momenta. The data for $D_{L}(p)$ are subject to severe finite- $N_{t}$ effects at $T \approx T_{c}$. As a result, only lattices with $N_{t} \geq 8$ seem to be free from systematic errors. After these errors are removed, we see an infrared value about $50 \%$ smaller than before. This suggests that there is no jump in the infrared value of $D_{L}(p)$ as $T \rightarrow T_{c}$ from below and that the drop after $T_{c}$ is significantly smaller than previously observed. [We note that all previous studies of $D_{L}(p)$ around $T_{c}$ have employed $N_{t} \leq 4$.] Therefore, the qualitative behavior of $D_{L}(p)$ around the transition has to be revised.

We have obtained good fits of our data to a Gribov-Stingl form, with comparable real and imaginary parts of the pole masses, also in the longitudinal-propagator case. This is in contrast with an electric screening mass defined by the expression $D_{L}(0)^{-1 / 2}$, which moreover may contain significant finite-size effects. In that respect, we plan to consider the upper and lower bounds for $D(0)$ introduced in [15], to investigate the infinite-volume limit of the gluon propagator at nonzero temperature.

\section{Acknowledgements}

The authors thank agencies Fapesp and CNPq for financial support. Our simulations were performed on the new CPU/GPU cluster at IFSC-USP (obtained through a Fapesp grant).

\section{References}

[1] A. D. Linde, Phys. Lett. B 96, 289 (1980); A. K. Rebhan, Phys. Rev. D 48, 3967 (1993).

[2] A. Cucchieri, F. Karsch and P. Petreczky, Phys. Rev. D 64, 036001 (2001).

[3] O. Oliveira and P. Bicudo, arXiv:1002.4151 [hep-lat].

[4] A. C. Aguilar, D. Binosi and J. Papavassiliou, Phys. Rev. D 78, 025010 (2008).

[5] A. Cucchieri and T. Mendes, to be submitted.

[6] D. Zwanziger, Nucl. Phys. B 364, 127 (1991).

[7] A. Cucchieri and T. Mendes, PoS QCD-TNT09, 026 (2009).

[8] A. Cucchieri, T. Mendes and A. R. Taurines, Phys. Rev. D 67, 091502 (2003).

[9] A. Cucchieri, T. Mendes and A. R. Taurines, Phys. Rev. D 71, 051902 (2005); P. O. Bowman et al., Phys. Rev. D 76, 094505 (2007).

[10] D. J. Gross, R. D. Pisarski and L. G. Yaffe, Rev. Mod. Phys. 53, 43 (1981).

[11] U. M. Heller, F. Karsch and J. Rank, Phys. Rev. D 57, 1438 (1998); A. Cucchieri, F. Karsch and P. Petreczky, Phys. Lett. B 497, 80 (2001).

[12] A. Cucchieri, A. Maas and T. Mendes, Phys. Rev. D 75, 076003 (2007).

[13] V. G. Bornyakov and V. K. Mitrjushkin, arXiv:1011.4790 [hep-lat].

[14] C. S. Fischer, A. Maas and J. A. Muller, Eur. Phys. J. C 68, 165 (2010).

[15] A. Cucchieri and T. Mendes, Phys. Rev. Lett. 100, 241601 (2008). 